\title{
Large Academic Hospital Laboratory Investigates a Major Pre-Analytical Challenge in Africa and Developing Countries
}

\author{
Ernest Philani Buthelezi ${ }^{1, ~ *}$, Florence Marule ${ }^{2}$, Bahule Nimrod Motlonye ${ }^{3}$, Ntsoaki Mopane ${ }^{4}$, \\ Tshepo Rakhothule ${ }^{2}$, Donald Moshen Tanyanyiwa ${ }^{5}$ \\ ${ }^{1}$ Department of Health, Johannesburg, South Africa \\ ${ }^{2}$ Department of Chemical Pathology, National Health Laboratory Service, Johannesburg, South Africa \\ ${ }^{3}$ National Health Laboratory Service, Johannesburg, South Africa \\ ${ }^{4}$ National Health Laboratory Service, Kalafong Hospital, Pretoria, South Africa \\ ${ }^{5}$ Department of Chemical Pathology, University of the Witwatersrand and National Health Laboratory Service, Johannesburg, South Africa
}

Email address:

Philani.Buthelezi@gauteng.gov.za (E. P. Buthelezi)

${ }^{*}$ Corresponding author

\section{To cite this article:}

Ernest Philani Buthelezi, Florence Marule, Bahule Nimrod Motlonye, Ntsoaki Mopane, Tshepo Rakhothule, Donald Moshen Tanyanyiwa. Large Academic Hospital Laboratory Investigates a Major Pre-Analytical Challenge in Africa and Developing Countries. Pathology and Laboratory Medicine. Vol. 3, No. 1, 2019, pp. 10-18. doi: 10.11648/j.plm.20190301.13

Received: December 20, 2018; Accepted: January 20, 2019; Published: February 7, 2019

\begin{abstract}
Background: Delay in serum separation from red blood cells in samples collected from most primary healthcare facilities and transported to a laboratory for analysis is of great concern. Standard guidelines state that serum or plasma should be separated from cells within 2 hours of collection. The aim was to determine effects of delayed sample separation on measured biochemical analytes. The objective was to store blood samples in primary collection tubes at $20-25^{\circ} \mathrm{C}$ post venesection, then separate, and analyse samples of selected analytes. Methods: Multiple sample tubes of whole blood were collected from one of the authors volunteer, and subjected to time delays in centrifugation. The baseline serum was separated from red blood cells within 30 minutes of post venesection to allow adequate coagulation. Twenty analytes were studied using 2 analytical platforms. Percentage variation and standard error method were used to evaluate time-dependent variability in analytes. Total change limit was used to measure significant changes within-run variability for both platforms. Results: Most analytes were stable up to day 3 to 4 on both platforms. Serum $\mathrm{CO}_{2}, \mathrm{CL}, \mathrm{Ca}$, ALT and ALB were stable up to 8 days when they were measured on Cobas $8000 \AA$. BUN, TRIG, TB, CHOL, AST, ALT and ALB were stable up to 10 days on Dimension $\AA$ CCS. K showed significant changes at $2 \mathrm{~h}$ on both platforms at initial measurements. It was out-of-range at day 10 on Dimension ${ }^{\circledR}$ CCS. Serum creatinine levels showed substantial changes at day 2 on Dimension ${ }^{\circledR}$ analyzer and on Cobas $8000 \AA$ at day 3. Conclusions: The study showed stability of wide range of serum analytes at $20-25^{\circ} \mathrm{C}$ for several days. The acceptable results can be achieved if samples are centrifuged the same day and analyzed later for most of biochemical analytes.
\end{abstract}

Keywords: Analyte, Delayed Measurement, Primary Healthcare, Analytical Platforms, Serum

\section{Introduction}

In laboratory medicine, the triad of pre-analytical, analytical and post-analytical stages play a crucial role in the production of accurate results, which physicians depend on for optimum patient management [1]. Recent studies on sources of error in laboratory medicine show that most errors occur at the pre-analytical stage [1-3]. The errors that occur translate to financial burden to the patient, laboratory and the hospital [3]. Therefore, key to providing precise, accurate and reliable test results is the pre-analytical sample storage temperature at phlebotomy site, transportation to a laboratory and at the laboratory [4-9]. Ideally, serum should be separated within 30 minutes of collection but an interval of $2 \mathrm{~h}$ is still acceptable to avoid increased activity of the red 
cells and transmembrane diffusion, which causes changes in the concentrations of serum analytes [4, 7-8, 10].

Chris Hani Baragwanath Academic Hospital (CHBAH) in the Gauteng province is one of the largest hospitals in the southern hemisphere. This hospital and the 50 primary healthcare facilities that filter into it, serves 52 suburbs. One of the biggest challenges is collection and delivery of samples from the surrounding clinics that filter through to the hospital. In an attempt to overcome this challenge, Johannesburg Health District and the National Health Laboratory Service agreed on a model to collect and transport samples in bulk from individual primary healthcare facilities to a central laboratory to ease healthcare costs. Often this takes 3 to $6 \mathrm{~h}$ to reach the laboratory, which is longer than the $2 \mathrm{~h}$ recommended in the WHO guidelines [4, $10]$.

The sample rejection by reason report, showed a very high percentage of blood samples being discarded as being 'too old' to be analyzed after 2-3 days. This was of great concern to the Department of Health because most patients using these healthcare facilities travel very long distances only to find out that their blood samples were rejected and to be requested for a second sample, which might be rejected again. On the other hand, delayed serum separation has serious impact on the accuracy of results due to the loss of the red cell membrane ability to optimally separate the intracellular and extracellular components apart at suboptimal temperature of $20-25^{\circ} \mathrm{C}$. Often the extraanalytical factors such as different transportation conditions, prolonged storage at high or low temperature, improper handling that may affect analysis process are not monitored. Previous studies showed that prolonged storage of whole blood samples lead to deterioration in stability of most analytes when subjected to different time-point measurements [4, 10-17]. Studies showed stability for a wide range of plasma analytes on blood samples kept at $21^{\circ} \mathrm{C}[7$, 17]. A study on the effect of temperature on 29 commonly measured analytes found that most of the serum analytes concentration were unaffected at temperatures of 30 to $38^{\circ} \mathrm{C}$ [4]. Another study of 81 analytes concluded that only a few routine analytes required stringent control of delivery times before serum-clot separation, with most routine biochemical tests tolerating long delays in transportation to the laboratory without changes in analyte content. Analysis of sera separated from clot within 30 minutes of collection and stored at freezing temperature showed stability on 16 out of 17 biochemical analytes.

Major concern was raised by clinicians following the 2017 South African HIV guidelines, which recommends the use of creatinine as a marker of renal function, to determine the suitability of using TDF based regimens. The concern from clinicians was based on suspiciously abnormal creatinine and eGFR results, which gave an impression of renal impairment. Confirmation of such results required recalling the patient; collect another blood sample for repeat testing or inappropriate referrals from nurse clinician within the facility to tertiary hospitals.
This is costly for both the patient and the Department of Health as it overburdens the hospitals. On the other hand, use of TDF in HIV patients with low eGFR is contraindicated [22].

Reliable serum creatinine measurements in glomerular filtration estimation are critical to the public sector [18], especially in South Africa with high burden of HIV and AIDS. In order for serum creatinine measurement to be clinically acceptable it must have a small total error for better estimation of eGFR of $<50 \mathrm{~mL} / \mathrm{min}$ [22]. A value greater than this value may signify presence of kidney disease. In the South African Antiretroviral Treatment Guideline (adult) 2017, all new patients are eligible for $\mathrm{TDF}+\mathrm{FTC}$ (or 3TC) + EFV (provided as fixed dose combination (FDC) [19]. Furthermore, understanding different methodologies of serum creatinine measurements may be important in ensuring limitations are taken into account when interpreting results [18, 20-21].

This study sought to evaluate the clinical implications of delayed separation of serum from the clot on routine biochemical measurements from commonly requested tests including creatinine. Creatinine is one of the most requested test at primary healthcare.

The aim was to determine effects of delayed sample separation and measurement of selected analytes on venepuncture collected blood stored at $20-25^{\circ} \mathrm{C}$.

\section{Design and Methods}

\subsection{Subject Selection}

On two different days, within the same week, multiple sample of whole blood in serum separator tubes (SST) were collected from the same subject. The collected samples were subjected to time delays in centrifugation intervals up to day 4. The same subject, same phlebotomist, same collection materials and the collection materials were used at the two sites. The duration of collection for each set of bloods was within 30 minutes to avoid any error between the first and last collected tube.

\subsection{Study Sites}

CHBAH-Chemical Pathology laboratory and Kalafong Provincial Tertiary Hospital laboratory.

\subsection{Equipment and Consumables}

The analytical platforms used were Cobas $8000 \AA$ from Roche Diagnostics SA at CHBAH laboratory and Dimension ${ }^{\circledR}$ Clinical Chemistry System (CCS) from Siemens S.A. Ltd at Kalafong laboratory. Becton Dickinson (BD) Vacutainer ${ }^{\mathrm{TM}}$ SST blood collection tubes were used for the study.

\subsection{Procedure}

Collected samples were stored in tube racks at $20^{-} 25^{\circ} \mathrm{C}$ prior and after analyses. Two different storing racks were 
used to separate processed and unprocessed sample tubes. On day 1 following centrifugation, one of the 20 samples was analyzed within $30 \mathrm{~min}$ to obtain the baseline value $\left(T_{C}\right)$. After the baseline was established the remaining 19 samples were each retrieved centrifuged and analyzed at different intervals according to the analysis schedule. After all the individual samples were analyzed, all the tubes were left for a few more day at $20^{-} 25^{\circ} \mathrm{C}$. On day 8 , without any further centrifugation, all 20 samples initially processed on Cobas $8000 \AA$ were loaded on the same rack and analyzed. On the other hand, on day 10, without any further centrifugation, all 20 samples initially processed on Dimension ${ }^{\circledR}$ were also loaded on the same rack and analyzed.

\subsection{Volunteer Data Collection}

Results of de-identified volunteer testing were recorded onto a MS Excel sheet, which was completed manually by the analyzer operator and collated to a central point. The aim of this agreed process was to enable the central point to tracking trends in serial analyte measurements across time.

\subsection{Data Analysis}

Data analysis was performed using MS Excel 2016 version. Imprecision was used as the performance indicator of analytical quality. Imprecision is expressed as a coefficient of variation ( $\mathrm{CV} \%)$, calculated using the formula: $\mathrm{CV} \%=$ (standard deviation of replicate $\mathrm{QC}$ measurements/mean of the replicate measurements) $* 100$. In simple terms, the lower the imprecision, the better the analytical performance of the device. The stability of the analyte was determined by calculating the percentage change of the (test value) results from the mean value $\left(T_{C}\right.$ value) at each time point and calculating the mean percentage change from each measurement as follows: $\left\{\left(\right.\right.$ Test $\left.\left.-\mathrm{T}_{\mathrm{C}}\right) / \mathrm{T}_{\mathrm{C}}\right\} * 100$. To establish outcome related analytical performance goals, the acceptable limits around 30-minutes values were derived from the analytical and intra-individual biological variation of the tests [22];

(a)The mean percentage deviation $(\% \Delta)$ was compared to acceptable change limit (ACL). The analytical imprecision (CVa), using formula $\mathrm{ACL}=2.77 \mathrm{CVa}$. The factor 2.77 is derived from $Z \sqrt{ } 2$, where $Z=1.96$, determined by $95 \%$ of confidence interval value for bidirectional changes, and $\mathrm{Z} \sqrt{2}$ as we are comparing two results with same CVa [23]. We used the CVa values from Royal College of Pathologists of Australia (RCPA) external quality assurance scheme (EQAS) to estimate probable differences in analyte concentration.

(b) To determine acceptable imprecision based on the intra-individual biological variation. The value of $\leq 0.5 \mathrm{CVb}$ was described [22, 24-26] and this value was used for each analyte. The biological variation was obtained from the European Federation Clinical Chemistry and Laboratory Medicine (EFLM) database.

(c) Mean differences of analytes were compared with the total change limit (TCL) which is calculated as: $\sqrt{ }(2.77$ $* \mathrm{CVa})^{2}+(0.5 * \mathrm{CVb})^{2}$; where $\mathrm{CVa}$ is analytical imprecision and $\mathrm{CVb}$ is intra-biological variation, $\mathrm{CVb}$ was taken from desirable specifications for inaccuracy $(\%)$.

\subsection{Ethics}

Study was done under the Wits Ethics approval for Evaluations studies of 2012 .

\section{Results}

Table 1. List of twenty analytes that were tested.

\begin{tabular}{|c|c|}
\hline 1 & Albumin (ALB) \\
\hline 2 & Alkaline Phosphatase (ALP) \\
\hline 3 & Alanine Transaminase (ALT) \\
\hline 4 & Aspartate Transaminase (AST) \\
\hline 5 & Calcium $(\mathrm{Ca})$ \\
\hline 6 & Carbon Dioxide $\left(\mathrm{CO}_{2}\right)$ \\
\hline 7 & Chloride (CL) \\
\hline 8 & Cholesterol (CHOL) \\
\hline 9 & Creatinine (CRT) \\
\hline 10 & Direct Bilirubin (DB) \\
\hline 11 & Gamma-Glutamyltransferase (GGT) \\
\hline 12 & High Density Lipoproteins (HDL) \\
\hline 13 & Inorganic Phosphorus $\left(\mathrm{PO}_{4}\right)$ \\
\hline 14 & Magnesium (Mg) \\
\hline 15 & Sodium $(\mathrm{Na})$ \\
\hline 16 & Potassium (K) \\
\hline 17 & Total Bilirubin (TB) \\
\hline 18 & Total Protein (TP) \\
\hline 19 & Triglycerides (TRIG) \\
\hline 20 & Urea (BUN) \\
\hline
\end{tabular}


Table 2. Analytical and biological variation for the tested analytes.

\begin{tabular}{|c|c|c|c|c|c|c|c|c|c|c|}
\hline \multirow{3}{*}{ Analyte } & \multicolumn{5}{|c|}{ Dimension ${ }^{\circledR}$ CCS } & \multicolumn{5}{|c|}{ Roche Cobas 8000(R) } \\
\hline & \multicolumn{2}{|c|}{ EQAS Analytical imprecision } & \multicolumn{3}{|c|}{ Sample analytical imprecision } & \multicolumn{2}{|c|}{ EQAS Analytical imprecision } & \multicolumn{3}{|c|}{ Sample analytical imprecision } \\
\hline & STDEV & $\mathrm{CV} \%$ & $\mathbf{T}_{\mathrm{C}}$ & CV\% 1-4 & CV\% 10 & STDEV & $\mathrm{CV} \%$ & $\mathbf{T}_{\mathrm{C}}$ & CV\% 1-4 & $\mathrm{CV} \% 8$ \\
\hline CRT & 5,41 & 8,4 & 83 & 2,23 & 3,28 & 4,70 & 2,8 & 81 & 9,6 & 13,2 \\
\hline $\mathrm{K}$ & 0,09 & 4,82 & 3,6 & 6,69 & 9,08 & 0,09 & 2 & 3,74 & 18,3 & 12,0 \\
\hline ALT & 1,57 & 15,5 & 25 & 2,76 & 5,22 & 13,40 & 6 & 18,6 & 4,8 & 5,8 \\
\hline ALB & 1,32 & 5,59 & 42 & 9,47 & 15,47 & 1,10 & 2,9 & 46,8 & 1,2 & 11,1 \\
\hline ALP & 1,53 & 12,91 & 51 & 3,63 & 5,34 & 13,40 & 6 & 51 & 1,7 & 2,2 \\
\hline DB & 1,56 & 25,7 & 2 & 33,24 & 28,34 & 1,80 & 3,3 & 3 & 8,7 & 13,2 \\
\hline TB & 1,75 & 11,34 & 5 & 29,17 & 35,36 & 1,80 & 3,5 & 5 & 6,5 & 11,3 \\
\hline $\mathrm{Ca}$ & 0,06 & 3,99 & 2,10 & 6,69 & 10,26 & 0,04 & 1,6 & 2,40 & 2,0 & 7,4 \\
\hline $\mathrm{Cl}$ & 3,18 & 3,74 & 99 & 0,75 & 6,86 & 8,90 & 39,5 & 25 & 1,5 & 1,5 \\
\hline $\mathrm{CO} 2$ & 1,38 & 14,84 & 25 & 7,46 & 30,28 & 1,40 & 1,5 & 17 & 3,7 & 8,8 \\
\hline GGT & 2,03 & 15,19 & 23 & 12,22 & 13,71 & 0,84 & 5,7 & 0,81 & 3,4 & 5,1 \\
\hline $\mathrm{Mg}$ & 0,03 & 4,78 & 0,82 & 10,28 & 15,54 & 0,08 & 5,7 & 1,03 & 3,3 & 2,6 \\
\hline $\mathrm{TP}$ & 2,08 & 5,2 & 83 & 2,14 & 3,33 & 0,09 & 2,3 & 4,2 & 1,4 & 2,4 \\
\hline BUN & 0,32 & 15,03 & 3,3 & 4,94 & 3,48 & 0,02 & 2,2 & 1,34 & 2,3 & 2,2 \\
\hline CHOL & 0,12 & 4,14 & 4,6 & 2,87 & 3,50 & 8,40 & 8,8 & 91,5 & 61,1 & 1,1 \\
\hline TRIG & 0,07 & 24,35 & 1,93 & 2,53 & 4,50 & 0,04 & 3,1 & 1,38 & 2,3 & 1,6 \\
\hline HDL & 0,04 & 6,2 & 1,29 & 2,85 & 4,35 & 2,00 & 1,5 & 132 & 70,6 & 1,7 \\
\hline
\end{tabular}

The analytical imprecision values (STDEV and CV\%) are shown for both Dimension ${ }^{\circledR}$ CCS and Roche Diagnostics $8000 \AA$, respectively. The imprecision values were obtained from the Thistle National External Quality Assurance System (EQAS).

Table 3. Percentage changes for the tested analytes on Roche Cobas $8000 ®$ analytical platform.

\begin{tabular}{|c|c|c|c|c|c|c|c|}
\hline & & $30 \mathrm{~min}$ & Day 1 & Day 2 & Day 3 & Day 4 & Acceptable \\
\hline Analyte & TCL & $\mathbf{T}_{\mathrm{C}}$ & $\% \Delta$ & $\% \Delta$ & $\% \Delta$ & $\% \Delta$ & time delay \\
\hline CRT & 8,0 & 81 & 3,70 & $-0,4$ & 3,1 & ${ }^{\mathrm{e}} \mathbf{4 0 , 7}$ & $72 \mathrm{~h}$ \\
\hline K & 5,6 & 3,74 & ${ }^{\mathrm{e}} 6,15$ & ${ }^{\mathrm{e}} 20,7$ & ${ }^{e} 38,3$ & ${ }^{\mathrm{e}} 102,4$ & $2 \mathrm{~h}$ \\
\hline ALT & 17,6 & 18,6 & 4,84 & $-0,2$ & 11,8 & 13,1 & $96 \mathrm{~h}$ \\
\hline ALB & 8,1 & 46,8 & 0,00 & 3,5 & 1,9 & 4,2 & $96 \mathrm{~h}$ \\
\hline ALP & 17,0 & 51 & 0,78 & $-1,1$ & 0,0 & 4,0 & $96 \mathrm{~h}$ \\
\hline AST & 10,0 & 31,7 & 2,46 & 1,6 & 8,9 & ${ }^{\mathrm{e}} 14,7$ & $72 \mathrm{~h}$ \\
\hline DB & 9,1 & 2,6 & 7,31 & $-2,7$ & ${ }^{\mathrm{e}} 11,5$ & ${ }^{\mathrm{e}}-10,8$ & $48 \mathrm{~h}$ \\
\hline TB & 10,7 & 5,2 & $-2,31$ & $-0,3$ & 3,4 & ${ }^{\mathrm{e}}-11,2$ & $72 \mathrm{~h}$ \\
\hline $\mathrm{Ca}$ & 4,5 & 2,4 & $-0,25$ & 1,2 & 2,5 & 3,8 & $96 \mathrm{~h}$ \\
\hline $\mathrm{Cl}$ & 24,4 & 91,5 & $-0,20$ & $-0,1$ & $-0,7$ & 1,4 & $96 \mathrm{~h}$ \\
\hline $\mathrm{CO}_{2}$ & 109,4 & 25,1 & $-2,15$ & $-10,0$ & $-9,6$ & $-19,1$ & $96 \mathrm{~h}$ \\
\hline GGT & 4,2 & 17 & ${ }^{\mathrm{e}}-4,71$ & 1,7 & ${ }^{\mathrm{e}} 10,3$ & ${ }^{\mathrm{e}} 4,7$ & $48 \mathrm{~h}$ \\
\hline $\mathrm{Mg}$ & 15,8 & 0,81 & 3,21 & 6,7 & 11,7 & ${ }^{\mathrm{e}} 24,9$ & $72 \mathrm{~h}$ \\
\hline $\mathrm{Na}$ & 4,2 & 132 & $-0,30$ & 1,2 & 1,1 & ${ }^{\mathrm{e}} 5,1$ & $72 \mathrm{~h}$ \\
\hline $\mathrm{PO}_{4}$ & 15,9 & 1,03 & $-2,14$ & $-2,5$ & ${ }^{\mathrm{e}} 85,0$ & ${ }^{\mathrm{e}} 315,1$ & $48 \mathrm{~h}$ \\
\hline TP & 4,8 & 77,3 & 0,65 & ${ }^{\mathrm{e}} 4,9$ & ${ }^{\mathrm{e}} 6,0$ & $-0,3$ & $24 \mathrm{~h}$ \\
\hline BUN & 6,5 & 4 & 0,00 & 2,9 & 4,4 & ${ }^{\mathrm{e}} 14,6$ & $72 \mathrm{~h}$ \\
\hline CHOL & 11,65 & 4,2 & $-0,57$ & ${ }^{\mathrm{e}} 226,1$ & 3,2 & NT & $24 \mathrm{~h}$ \\
\hline TRIG & 1,93 & 1,38 & $-1,88$ & $-0,9$ & $-0,2$ & NT & $48 \mathrm{~h}$ \\
\hline HDL & 1,29 & 1,34 & $-0,30$ & e 369,8 & 2,4 & NT & $24 \mathrm{~h}$ \\
\hline
\end{tabular}

The analysis of acceptable change limits (ACL) on all analytes measured on Roche Cobas $8000 ®$ analyzer. Analytes were subjected to different time delay measurement intervals up to 4 days. Total change limits (TCL) and acceptable time delays are shown. \% $\%$ - percentage variation; NT- not tested.

Table 4. Percentage changes of tested analytes between day 1-4 and repeat measurements at day 8 using Cobas 8000 ( analyzer.

\begin{tabular}{|c|c|c|c|c|c|c|c|c|}
\hline \multirow{2}{*}{ Testing days } & \multirow{2}{*}{ Analyte } & \multirow{2}{*}{ TCL } & $30 \mathrm{~min}$ & Day 1 & Day 2 & Day 3 & Day 4 & Acceptable \\
\hline & & & $\mathbf{T}_{\mathbf{C}}$ & $\% \Delta$ & $\% \Delta$ & $\% \Delta$ & $\% \Delta$ & time delay \\
\hline Day 1-4 & TP & 4,8 & 77 & 0,65 & 4,9 & 6,0 & $-0,3$ & $48 \mathrm{~h}$ \\
\hline Day 8 & TP & 4,8 & 75 & 5,68 & 6,76 & 7,45 & 9,63 & $24 \mathrm{~h}$ \\
\hline Day 1-4 & TB & 10,7 & 5,2 & $-2,31$ & $-0,3$ & 3,4 & $-11,2$ & $72 \mathrm{~h}$ \\
\hline Day 8 & TB & 10,7 & 4,4 & 0,57 & 7,27 & 14,20 & 20,45 & $72 \mathrm{~h}$ \\
\hline Day 1-4 & PO4 & 15,9 & 1,03 & $-2,14$ & $-2,5$ & 85,0 & 315,1 & $72 \mathrm{~h}$ \\
\hline Day 8 & PO4 & 15,9 & 1,18 & $-6,36$ & $-16,61$ & 130,93 & 317,80 & $48 \mathrm{~h}$ \\
\hline Day 8 & $\mathrm{Na}$ & 4,2 & 134 & 1,70 & 1,54 & 2,81 & 4,64 & $72 \mathrm{~h}$ \\
\hline Day 1-4 & $\mathrm{Mg}$ & 15,8 & 0,81 & 3,21 & 6,7 & 11,7 & 24,9 & $72 \mathrm{~h}$ \\
\hline
\end{tabular}




\begin{tabular}{|c|c|c|c|c|c|c|c|c|}
\hline \multirow{2}{*}{ Testing days } & \multirow{2}{*}{ Analyte } & \multirow{2}{*}{ TCL } & \multirow{2}{*}{$\begin{array}{l}30 \text { min } \\
T_{C} \\
\end{array}$} & \multirow{2}{*}{$\begin{array}{l}\text { Day } 1 \\
\% \Delta\end{array}$} & \multirow{2}{*}{$\begin{array}{l}\text { Day } 2 \\
\% \Delta\end{array}$} & \multirow{2}{*}{$\begin{array}{l}\text { Day } 3 \\
\% \Delta\end{array}$} & \multirow{2}{*}{$\begin{array}{l}\text { Day } 4 \\
\% \Delta\end{array}$} & \multirow{2}{*}{$\begin{array}{l}\text { Acceptable } \\
\text { time delay }\end{array}$} \\
\hline & & & & & & & & \\
\hline Day 8 & $\mathrm{Mg}$ & 15,8 & 0,81 & 4,01 & 7,90 & 14,81 & 22,84 & $72 \mathrm{~h}$ \\
\hline Day 1-4 & $\mathrm{K}$ & 5,6 & 3,74 & 6,15 & 20,7 & 38,3 & 102,4 & $2 \mathrm{~h}$ \\
\hline Day 8 & $\mathrm{~K}$ & 5,6 & 3,90 & 11,35 & 26,41 & 57,24 & 115,13 & $2 \mathrm{~h}$ \\
\hline Day 1-4 & GGT & 6,9 & 17 & $-4,71$ & 1,7 & 10,3 & 4,7 & $48 \mathrm{~h}$ \\
\hline Day 8 & GGT & 6,9 & 15 & 13,33 & 12,00 & 18,33 & 20,00 & $48 \mathrm{~h}$ \\
\hline Day 1-4 & $\mathrm{DB}$ & 11,6 & 3 & 7,31 & $-2,7$ & 11,5 & $-10,8$ & $96 \mathrm{~h}$ \\
\hline Day 8 & DB & 11,6 & 2 & 5,00 & 8,00 & 26,25 & 32,50 & $72 \mathrm{~h}$ \\
\hline Day 1-4 & CRT & 8,0 & 81 & 3,70 & $-0,4$ & 3,1 & 40,7 & $72 \mathrm{~h}$ \\
\hline Day 8 & CRT & 8,0 & 77 & 7,14 & $-2,34$ & 22,60 & 32,01 & $48 \mathrm{~h}$ \\
\hline Day 1-4 & $\mathrm{CO} 2$ & 109,4 & 25 & $-2,15$ & $-10,0$ & $-9,6$ & $-19,1$ & $96 \mathrm{~h}$ \\
\hline Day 8 & $\mathrm{CO} 2$ & 109,4 & 22 & $-3,01$ & $-8,21$ & $-16,29$ & $-21,21$ & $96 \mathrm{~h}$ \\
\hline Day 1-4 & $\mathrm{Cl}$ & 24,4 & 92 & $-0,20$ & $-0,1$ & $-0,7$ & 1,4 & $96 \mathrm{~h}$ \\
\hline Day 8 & $\mathrm{Cl}$ & 24,4 & 94 & $-0,11$ & $-2,44$ & $-5,74$ & $-7,49$ & $96 \mathrm{~h}$ \\
\hline Day 1-4 & $\mathrm{Ca}$ & 4,5 & 2,40 & $-0,25$ & 1,2 & 2,5 & 3,8 & $96 \mathrm{~h}$ \\
\hline Day 8 & $\mathrm{Ca}$ & 4,5 & 2,45 & 0,10 & $-7,18$ & 2,04 & 1,02 & $96 \mathrm{~h}$ \\
\hline Day 1-4 & BUN & 6,4 & 4.0 & 0,00 & 2,9 & 4,4 & 14,6 & $72 \mathrm{~h}$ \\
\hline Day 8 & BUN & 6,4 & 3,9 & 1,92 & 5,13 & 8,97 & 12,82 & $24 \mathrm{~h}$ \\
\hline Day 1-4 & AST & 10,0 & 32 & 2,46 & 1,6 & 8,9 & 14,7 & $72 \mathrm{~h}$ \\
\hline Day 8 & AST & 10,0 & 32 & 5,68 & 10,79 & 15,22 & 30,60 & $72 \mathrm{~h}$ \\
\hline Day 1-4 & ALT & 17,6 & 19 & 4,84 & $-0,2$ & 11,8 & 13,1 & $96 \mathrm{~h}$ \\
\hline Day 8 & ALT & 17,6 & 18 & $-3,57$ & 2,31 & 13,32 & 35,16 & $96 \mathrm{~h}$ \\
\hline Day 1-4 & ALP & 17,0 & 51 & 0,78 & $-1,1$ & 0,0 & 4,0 & $96 \mathrm{~h}$ \\
\hline Day 8 & ALP & 17,0 & 51 & 1,47 & 1,18 & 2,45 & 2,94 & $72 \mathrm{~h}$ \\
\hline Day 1-4 & ALB & 8,1 & 47 & 0,00 & 3,5 & 1,9 & 4,2 & $96 \mathrm{~h}$ \\
\hline Day 8 & ALB & 8,1 & 48 & 0,00 & $-12,00$ & 1,49 & 3,71 & $96 \mathrm{~h}$ \\
\hline
\end{tabular}

The list of all analytes for day 1-4 and day 8 are listed in column 2 with the corresponding analytical imprecision measurements on the same rows as analytes. TCL for all analytes and acceptable time delays are shown.

Table 5. Percentage changes for the tested analytes on Siemens Dimension ${ }^{\circledR}$ CCS analytical platform.

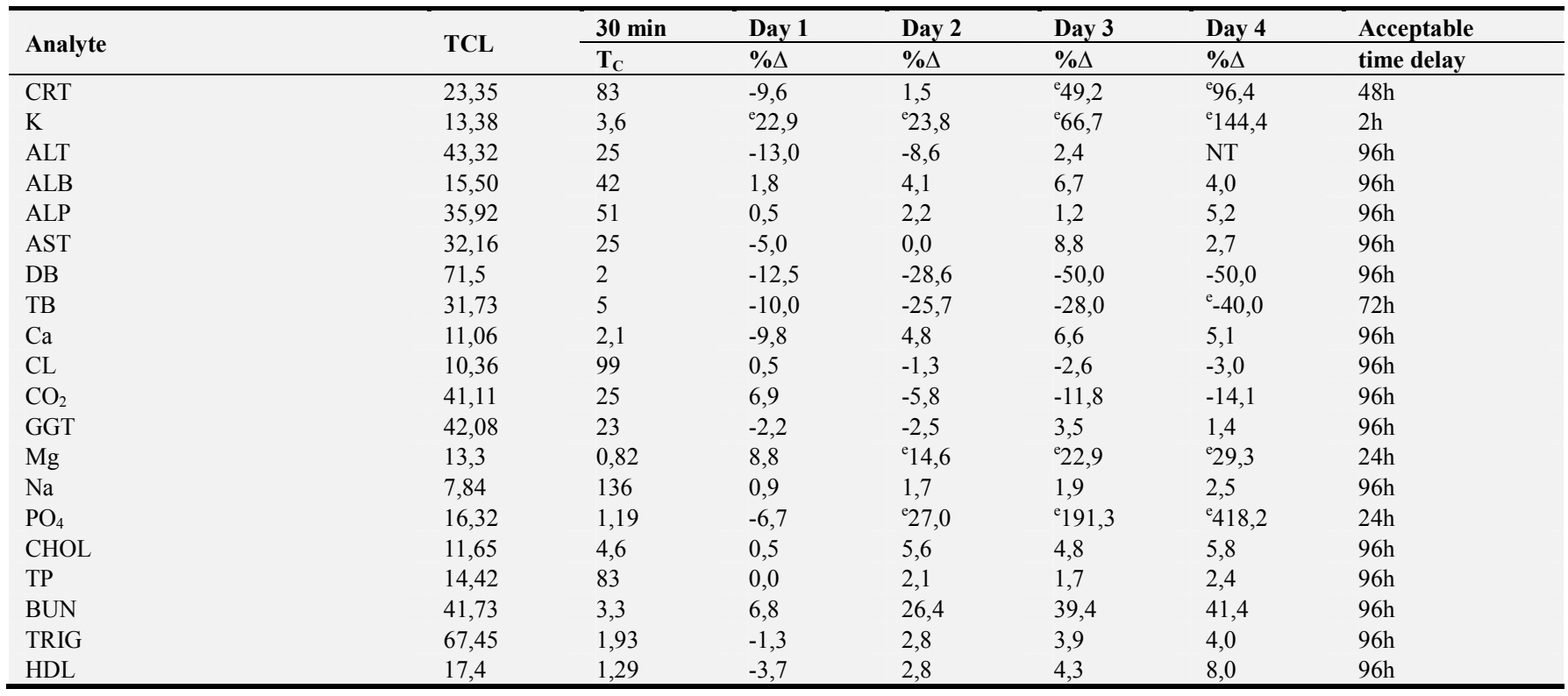

The analysis of acceptable change limits (ACL) on all analytes measured on Dimension ${ }^{\circledR}$ CCS analyzer. Analytes were subjected to different time delay measurement intervals up to 4 days. Total change limits (TCL) and acceptable time delays are shown. \% $\%$ - percentage variation; NT- not tested.

Table 6. Percentage changes of tested analytes between day 1-4 and repeat measurements at day 10 using Dimension ${ }^{\circledR}$ CCS analyzer.

\begin{tabular}{|c|c|c|c|c|c|c|c|c|}
\hline \multirow{2}{*}{ Testing days } & \multirow{2}{*}{ Analyte } & \multirow{2}{*}{ TCL } & 30 min & Day 1 & Day 2 & Day 3 & Day 4 & Acceptable \\
\hline & & & $\mathbf{T}_{\mathrm{C}}$ & $\% \Delta$ & $\% \Delta$ & $\% \Delta$ & $\% \Delta$ & time delays \\
\hline Day 1-4 & Urea & 41,7 & 3,3 & 6,8 & 26,4 & 39,4 & 41,4 & $96 \mathrm{~h}$ \\
\hline Day 10 & Urea & 41,7 & 4 & 7,5 & 12,1 & 17,5 & 27,5 & $96 \mathrm{~h}$ \\
\hline Day 1-4 & TRIG & 67,6 & 1,93 & $-1,3$ & 2,8 & 3,9 & 4 & $96 \mathrm{~h}$ \\
\hline Day 10 & TRIG & 67,6 & 2,15 & 6,2 & 5,8 & 4,2 & 0,2 & $96 \mathrm{~h}$ \\
\hline Day 1-4 & TB & 31,7 & 5 & -10 & $-25,7$ & -28 & -40 & $72 \mathrm{~h}$ \\
\hline Day 10 & TB & 31,7 & 1 & 0 & 0 & 100 & 100 & O-O-R \\
\hline
\end{tabular}




\begin{tabular}{|c|c|c|c|c|c|c|c|c|}
\hline \multirow{2}{*}{ Testing days } & \multirow{2}{*}{ Analyte } & \multirow{2}{*}{ TCL } & \multirow{2}{*}{$\begin{array}{l}30 \text { min } \\
T_{C} \\
\end{array}$} & \multirow{2}{*}{$\begin{array}{l}\text { Day } 1 \\
\% \Delta \\
\end{array}$} & \multirow{2}{*}{$\begin{array}{l}\text { Day } 2 \\
\% \Delta \\
\end{array}$} & \multirow{2}{*}{$\begin{array}{l}\text { Day } 3 \\
\% \Delta \\
\end{array}$} & \multirow{2}{*}{$\begin{array}{l}\text { Day } 4 \\
\% \Delta\end{array}$} & \multirow{2}{*}{$\begin{array}{l}\text { Acceptable } \\
\text { time delays }\end{array}$} \\
\hline & & & & & & & & \\
\hline Day 1-4 & TP & 14,4 & 83 & 0 & 2,1 & 1,7 & 2,4 & $96 \mathrm{~h}$ \\
\hline Day 10 & $\mathrm{TP}$ & 14,4 & 84 & 4 & 7,1 & 11,1 & 6,3 & $96 \mathrm{~h}$ \\
\hline Day 1-4 & $\mathrm{Na}$ & 7,8 & 136 & 0,9 & 1,7 & 1,9 & 2,5 & $96 \mathrm{~h}$ \\
\hline Day 10 & $\mathrm{Na}$ & 7,8 & 141 & 0,9 & $-3,7$ & 0,5 & $-8,5$ & $72 \mathrm{~h}$ \\
\hline Day 1-4 & K & 13,4 & 3,6 & 22,9 & 23,8 & 66,7 & 144,4 & $2 \mathrm{~h}$ \\
\hline Day 10 & $\mathrm{~K}$ & 13,4 & 5 & 0,7 & $-3,7$ & 46,7 & 80,7 & O-O-R \\
\hline Day 1-4 & $\mathrm{PO}_{4}$ & 16,3 & 1,19 & $-6,7$ & 27 & 191,3 & 418,2 & $24 \mathrm{~h}$ \\
\hline Day 10 & $\mathrm{PO}_{4}$ & 16,3 & 1,56 & $-11,5$ & 14 & 190,2 & 285,7 & O-O-R \\
\hline Day 1-4 & $\mathrm{Mg}$ & 13,3 & 0,82 & 8,8 & 14,6 & 22,9 & 29,3 & $48 \mathrm{~h}$ \\
\hline Day 10 & $\mathrm{Mg}$ & 13,3 & 0,93 & 2,9 & 5 & 10,8 & 7,9 & O-O-R \\
\hline Day 1-4 & HDL & 17,4 & 1,29 & $-3,7$ & 2,8 & 4,3 & 8 & $96 \mathrm{~h}$ \\
\hline Day 10 & HDL & 17,4 & 1,34 & 0,7 & 10,4 & 19,4 & 17,2 & O-O-R \\
\hline Day 1-4 & GGT & 42,4 & 23 & $-2,2$ & $-2,5$ & 3,5 & 1,4 & $96 \mathrm{~h}$ \\
\hline Day 10 & GGT & 42,4 & 18 & 14,8 & 7,4 & 18,5 & 35,2 & O-O-R \\
\hline
\end{tabular}

The list of all analytes for day 1-4 and day 10 are listed in column 2 with the corresponding analytical imprecision measurements on the same rows as analytes. TCL for all analytes and acceptable time delays are shown. $\% \Delta$ - percentage variation; NT- not tested.

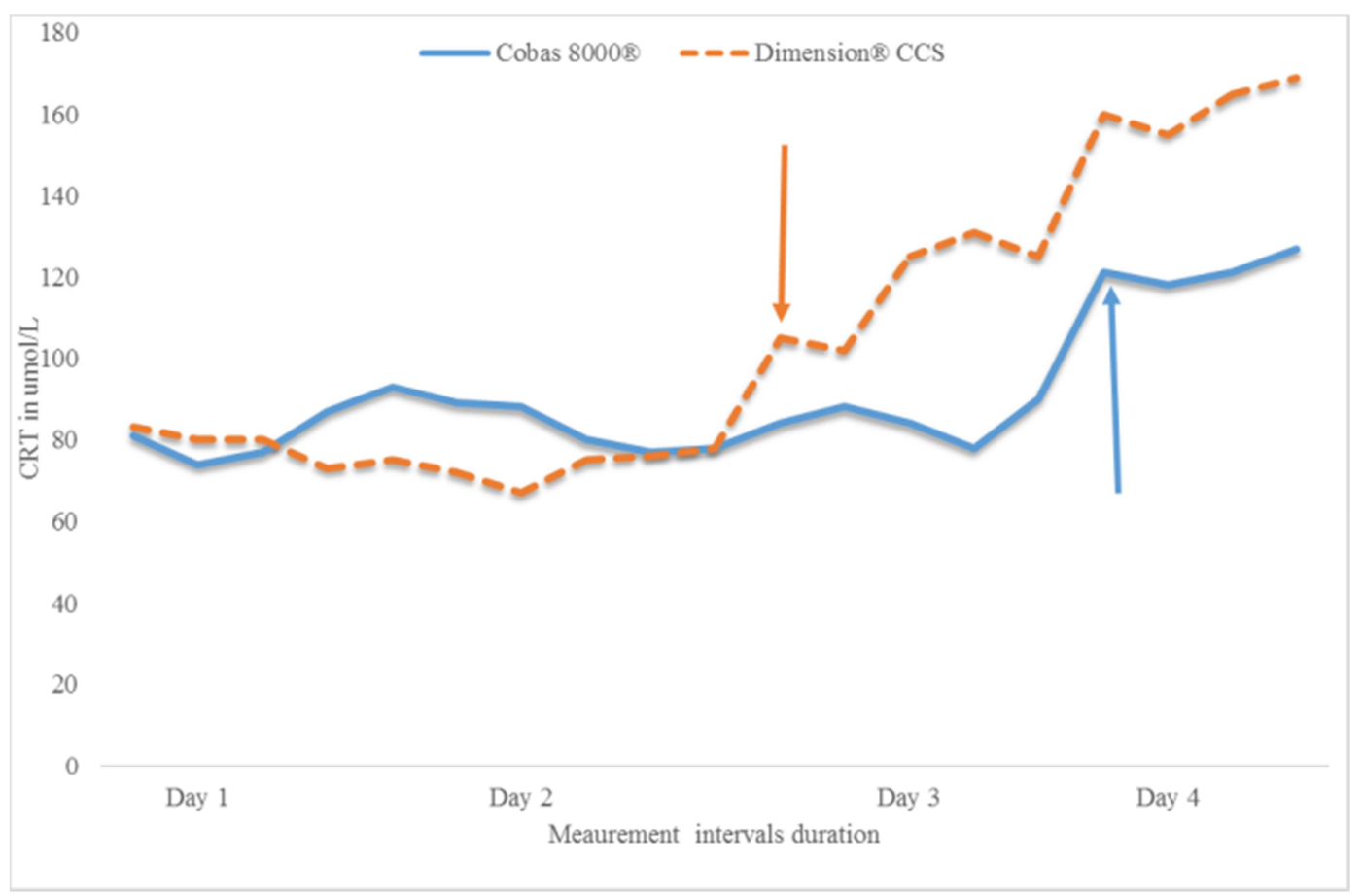

Figure 1. Graphic demonstration of TCL between Cobas $8000 ®$ and Dimension ${ }^{\circledR}$ CCS day 1 to 4 .

\section{Discussion}

This study was undertaken to investigate the effects of delayed centrifugation have on subsequent measurements of twenty analytes on two different analytical platforms. The objective was to assess the time-delayed serum separation on creatinine measurement on venepuncture collected blood kept at $20-25^{\circ} \mathrm{C}$.

Most analytes were stable up to day 3 to 4 (72-96h) on both platforms. This study confirms findings in another study where whole blood samples stored at $20-25^{\circ} \mathrm{C}$ (room temperature) for 1,2,3,4,8,24 and 48 hours and the percentage change for each result at 2-48 hours were calculated.

$\mathrm{PO}_{4}$ showed significant changes at $48 \mathrm{~h}$ on Cobas and $24 \mathrm{~h}$ on Dimension ${ }^{\circledR}$ CCS and out-of-range at day 10. Serum CRT levels showed substantial changes at day 2 on Dimension ${ }^{\circledR}$ analyzer and on Cobas $8000 \AA$ at day 3. Dimension ${ }^{\circledR}$ CCS results confirm Rehak and Chiang findings using whole blood stored at $3,10,15,22,25$ and $38^{\circ} \mathrm{C}$ on these analytes but different results with AST and ALT [4].

Inorganic phosphorus changes at $48 \mathrm{~h}$ on Cobas $8000 \AA$ and at $24 \mathrm{~h}$ on Dimension ${ }^{\circledR}$ CCS. Eighteen out of twenty studied analytes showed analyte stability beyond 24 hours on both platforms. This is in agreement with previous studies [4, 1718]. In another study using different storage temperatures, smallest changes in concentration was found at $20-25^{\circ} \mathrm{C}$ [4].

$\mathrm{K}$ and $\mathrm{PO}_{4}$ were respectively sensitive to delay in serum separation and significant changes were observed at $2 \mathrm{~h}$ (Cobas $8000 \AA$, Dimension ${ }^{\circledR}$ CCS); 48h (Cobas $\left.8000 \AA\right)$ ), 24h (Dimension ${ }^{\circledR}$ CCS). The remainder of the analytes were stable up to day 3 (72h) and beyond day 4 (96h) (tables 3 and 5). This is in agreement with previous studies [13-14], but different from the study by Zhang et al which reported 
significant changes at $3 \mathrm{~h}$ for $\mathrm{K}$ and $\mathrm{CL}$ at $8 \mathrm{~h}$ with other tests stable up to $48 \mathrm{~h}$. Changes in potassium are due to $\mathrm{Na}^{+} / \mathrm{K}^{+}$ ATPase pump, resulting in extracellular leak through passive transport and is well reported in textbooks [4].

Serum $\mathrm{CO}_{2}, \mathrm{CL}, \mathrm{Ca}, \mathrm{ALT}$ and ALB were stable up to 8 days when they were measured on Cobas $8000 \AA$. Urea, TRIG, TB, CHOL, AST, ALT and ALB were stable up to 10 days on Dimension ${ }^{\circledR}$ CCS. K was most sensitive to delayed separation of serum from clot on both platforms with significant changes observed at $2 \mathrm{~h}$ initial measurements and at day 8 on Cobas $8000 \AA$ whereas at day 10 it was out-ofrange on Dimension ${ }^{\circledR}$ CCS (tables 4 and 6).

There were no significant changes on $\left(\mathrm{PO}_{4}, \mathrm{Na}, \mathrm{Mg}\right.$, GGT, CRT, $\mathrm{CO}_{2}, \mathrm{CL}, \mathrm{Ca}, \mathrm{AST}$, ALT and ALB), between day 1-4 and day 8 interval measurements (table 4). Changes were observed on TP (48-24h), Urea (72-24h), ALP (96-72h) On day 8 for Cobas $8000 \AA$, most analytes were within total change limits (TCL) up to day 4; CRT up to day 3 (72h), $\mathrm{PO}_{4}$ up to day $2(48 \mathrm{~h})$ and $\mathrm{K}$ at $2 \mathrm{~h}$.

Between day 1-4, and day 10 twenty analytes were measured on Dimension ${ }^{\circledR}$ CCS. On day 10 , fifty percent of measured analytes were out-of-range (TB, $\mathrm{K}, \mathrm{PO}_{4}, \mathrm{Mg}$, HDL, GGT, DB, $\mathrm{CO}_{2}$ and $\mathrm{Ca}$ ). BUN, TRIG, TP, CHOL, AST, ALP, ALB were stable up to day 4 (96h). On day 1-4 concentrations were reliable up to day $3(72 \mathrm{~h})$ and at day 10 , $\mathrm{Na}$ and CL concentrations were stable up to day 4 (96h). ALT was measured up to day $3(72 \mathrm{~h})$ at day 10 due to insufficient sample available. CRT was stable up to day 2 (48h) at both 14 and day 10 measurements. Discrepancies in concentrations were observed on TP, $\mathrm{K}, \mathrm{PO}_{4}, \mathrm{Mg}$, HDL, GGT, $\mathrm{CO}_{2}$, and $\mathrm{Ca}$ when day 1-4 and day 10 measurement intervals were compared (table 6). This may be due to the methodology used for measurement of these analytes.

Temperature sensitive significant changes were observed on creatinine, glucose, inorganic phosphorus, potassium, AST and ALT on stored whole blood at 3, 10, 15, 22, 25, 30 and $38^{\circ} \mathrm{C}$ for $24 \mathrm{~h}$ before serum-clot separation $[4,10,18,20]$. In this study CRT was stable up to day 2 on Dimension ${ }^{\circledR}$ CCS at day 1-4 and day 10; and, up to day 3 on Cobas $8000 ®$ platform. This is not in agreement with another study that showed stability up to a week of most analytes including creatinine [18]. Differences observed may be due to different measurement methods and statistical analysis used.

CRT showed changes at day 2 on the Dimension ${ }^{\circledR}$ CCS and on Cobas $8000 \AA$ the significant change was observed at day 3. The discrepancy may reflect differences in analytical methods used by Roche Cobas $8000 \AA$ and the Dimension ${ }^{\circledR}$ CCS. Modifications in analytical measurements may be the cause of the differences observed. Clinically it is desirable that serum creatinine measurements have a small enough total error that the impact on the total uncertainty of estimated glomerular filtration rate (GFR) remains within clinically acceptable limits. Baseline value from laboratory must be $>50 \mathrm{~mL} / \mathrm{min}$. The consequence of incorrectly high CRT results reported pose a dilemma to a clinician as they reflect renal pathology which may not be there if the sample was analyzed on day one. Clinicians faced with this dilemma usually repeat test. This entails recalling a patient who may be already incapacitated for another blood to be taken. The sample may be analyzed on the same day giving different result from the previous sample. This creates doubts on the quality of the results and makes the client unhappy. If the client sample was analyzed on day 1 , ARV first line treatment would be initiated.

The timing of serum separation from the clot has implications on routine CRT measurements for clients to be put on comprehensive care, management and treatment of HIV and AIDS (CCMT) South Africa program and monitoring those who are already on the program. The study has demonstrated that the concentrations of CRT can be measured reliably in samples stored at $20-25^{\circ} \mathrm{C}$ for at least 3 days after centrifugation on Cobas $8000 \AA$ and up to 2 days on Dimension ${ }^{\circledR}$ CCS. The conclusion to the previous stability studies have been difficult to interpret due to variety of study designs, type of subjects from whom samples were collected, different analytical platforms and different statistical tools; but confirms findings from other timedependent variability of biochemical stability studies [21]. Similar to other reports, the results of this study have provided justification to make optimum decisions regarding sample stability prior to analysis [14]. K could be used as an indicator whether whole blood centrifugation had been delayed for extended period [6].

The findings from the study were used to create an evidence-based policy recommending centrifugation within a specific period upon arrival in the laboratory. The policy resulted in a reduction from $88 \%$ to less than $30 \%$ of samples rejected as "too old" for analysis. These results are a confirmation that the intervention is working.

\section{Conclusions}

Centrifugation of samples in SST tubes to separate serum from clot within 60 minutes improves stability of analytes present in serum. The study also concludes that after centrifugation samples can be stored at ambient temperature.

\section{Recommendations}

Placement of centrifuges at healthcare facilities that serve as specimen depots is strongly recommended in order to provide quality results even if there are delays in transportation of samples to central laboratories. After centrifugation, the samples must be kept closed in their primary tubes and stored at ambient temperature until analysis.

\section{Strength and Weaknesses}

This is the first study carried out at both the largest teaching academic hospital laboratory and a small to medium district laboratory under similar conditions.

This is the first study to investigate stability of analytes on samples obtained from a single donor. It is also the first study 
to investigate analytes on two separate analytical platforms.

An evaluation of a few samples stored at $4^{0} \mathrm{C}$ would have been helpful to evaluate the effects of refrigeration of blood samples awaiting collection.

\section{Acknowledgements}

Special appreciation and gratitude is extended Dr Ammina Ditta for carrying out the first pilot study on sample stability in the department, Dr Mpho Maphayi with a follow-up study that assessed the impact on serum samples left uncapped at ambient environment and the Business Managers for Chris Hani Baragwanath $\mathrm{AH}$ and Kalafong laboratory.

\section{References}

[1] Tiwari, E., Pallipady, A. and Mishra, S. 2015. Preanalytical, Analytical and Postanalytical Errors in Chemical Laboratory. International Journal of Science and Research, 4 (3): p22792281 .

[2] Plebani, M. 2006. Errors in clinical laboratories or errors in laboratory medicine. Clinical Chemistry and Laboratory Medicine, 44: p750-9.

[3] Green, S. F. 2013. The cost of poor blood specimen quality and errors in pre-analytical processes. Clinical Biochemistry, 46: p1175-9.

[4] Rehak, N. N. and Chiang, B. T. 1988. Storage of whole blood: Effect on the measured concentration of analytes in serum. Clinical Chemistry, 34/10: p2111-2114.

[5] Tanner, M., Kent, N., Smith, B., Fletcher, S. and Lewer, M. 2008. Stability of common biochemical analytes in serum gel tubes subjected to various storage temperatures and times precentrifugation. Annals of Clinical Biochemistry, 45: p375-379.

[6] Kalasker, V. and Sudhhamadhuri, A. 2015. Effect of serum clot contact time as a major source of preanalytical variation in serum electrolytes. International Journal of Research in Health Sciences, 3: p2278-281.

[7] Henriksen, L. O., Faber, N. R. and Moller, M. F. 2014. Stability of 35 biochemical and immunological routine tests after 10hours storage and transport of human whole blood at 210C. Scandinavian Journal of Clinical and Laboratory Investigation, 74: p603-10.

[8] Quartey, P., Quartey, P., James, O-T. and Yawo, S. R. 2018. Stability of selected biochemical analytes in plasma samples stored under different time and temperature conditions. Journal of Clinical Chemistry and Laboratory Medicine, 1 (2): p1-4.

[9] Pahwa, M. B., Menaka, K., Manish Raj, M. and Singh, V. 2015. Effect of storage time and temperature on serum clinical biochemistry analytes. Biochemistry: An Indian Journal, 9/4, p150-156.

[10] Baruah, A., Goyal, P., Sinha, S., Sinha, S., Ramesh, K. L. and Datta, R. 2014. Delay in processing - Major source of preanalytical variation in serum analytes. Journal of Clinical and Diagnostic Research, 8(12): pCC01-CC03.

[11] Vernekar, N. V. and Jabannavar, V. B. 2017. Effect of storage and temperature on two biochemical analytes (creatinine and urea) in pooled serum samples stored at $-20^{\circ} \mathrm{C}$. Indian Journal of Health Sciences and Biomedical Research, 10: p63-7.

[12] Wu, D. W., Li, Y. M. and Wang, F. 2017. How long can we store blood samples: a systematic review and meta-analysis EBioMedicine, 24: p277-285.

[13] Laessig, R. H., Indricksson, A. A., Hassemer, D. J., Paskey, T. A. and Schwartz, T. H. 1976. Changes in serum chemical values as a result of prolonged contact with the clot. American Journal of Clinical Pathology, 66: p598-604.

[14] Heins, M., Heil, W. and Withold, W. 1995. Storage of serum or whole blood samples? Effect of time and temperature on 22 serum analytes. European Journal of Clinical Chemistry and Clinical Biochemistry, 33: p231-238.

[15] Kachlawa, K., Kachlawa, P., Varma, M., Behera. R., Agrawal, D. and Kumar, S. 2017. Study of the stability of various biochemical analytes in samples stored at different predefined storage conditions at an accredited laboratory in India. Journal of Laboratory Physicians. 1: p11-15.

[16] Lippi, G., Guidi, G. C., Mattiuzi, C. and Plebani, M. 2006. Preanalytical variability: the dark side of the moon in laboratory testing. Clinical Chemistry and Laboratory Medicine, 44: p358-65.

[17] Clark, S., Youngman, L. D., Palmer, A., Parish, S., Peto, R. and Collins, R. 2003. Stability of plasma analytes after delayed separation of whole blood: implications for epidemiological studies. International Journal of Epidemiology, 32: p125-130.

[18] Cuhadar, S., Atay, A., Koseoglu, M., Dirican, A. and Hur, A. 2012. Stability of common biochemical analytes in serum separator tubes with or without gel barrier subjected to various storage conditions. Biochemica Medica, 22 (2): p202-14.

[19] Myers, G. L., Miller, W. G., Coresh, J., Fleming, J., Greenberg, N., Greene, T., Hostetter, T., Levey, A. S. Panteghini, M., Welch, M. and Eckfeldt, J.H. 2006. Recommendations for improving serum creatinine measurement: A report from the Laboratory Working Group of the National Kidney Disease Education Program. Clinical Chemistry, 52: p15-18.

[20] Shepherd, J., Warner, M. H. and Kilpatrick, E. S. 2007. Stability of creatinine with delayed separation of whole blood and implications for eGFR. Annals of Clinical Biochemistry, 44: p384-387.

[21] Joffe, M., Hsu, C. Y., Feldman, H. I., Weir, M., Landis, J. R. and Hamm, L. L. 2010. Variability of creatinine measurements in clinical laboratories: results from the. Chronic Renal Insufficiency Cohort (CRIC) Study Group American journal of nephrology, 31(5): p426-434.

[22] Fraser, C. G., Petesen, P. H., Ricos, C. and Haeckel, R. 1992. Proposed quality specifications for the imprecision and inaccuracy of analytical systems for clinical chemistry. European Journal of Clinical Chemistry and Clinical Biochemistry. 30(5): p311-7.

[23] Ricos, C., Alvarez, V., Cava, F., Garcia-Lario, J.V., Hernandez, A. and Jimenez, C.V., Minchinela, J., Perich, C. and Simon, M. 1999. Current databases on biologic variation; pros, cons and progress. Scandinavian Journal of Clinical and Laboratory Investigation, 59: p494-500. 
[24] Ricos, C., Alvarez, V., Cava, F., Garcia-Lario, J. V., Hernandez, A. and Jimenez, C. V., Minchinela, J., Perich, C. and Simon, M. 2009.Desirable quality specifications for total error, imprecision, and bias, derived from biological variation. http:/www.Westgard.com/biodatabase1.htm. Accessed 5 December 2018.

[25] Westgard, J. O., Seehafer, J. J. and Barry, P. L. 1994. European specification for imprecision and inaccuracy compared with operating specifications that assure the quality required by US CLIA Proficiency-Testing criteria. Clinical Chemistry, 40 (7): p1228-1232.

[26] Westgard James Quality requirements: Desirable biological variation database specifications. https://www.westgard.com/biodatabase1.htm. Accessed 5 December 2018. 